\title{
Überlegungen zur Aussprachebewusstheit im Kontext der Fremdsprachenvermittlung
}

\section{Einleitung}

Das Ziel des Ausspracheunterrichts ist vor allem die weitgehende Eliminierung des fremden Akzents. Zu diesem Zweck sollten die jeweils gegebenen sprachsystembedingten Unterschiede zwischen $\mathrm{L}_{1}$ und $\mathrm{L}_{2}$ in der Artikulation und Prosodie aufgezeigt sowie diesbezügliche funktionale Regularitäten in der Zielsprache unter Anwendung verschiedener Ausspracheübungen trainiert werden. ${ }^{1}$ Zugleich sollte es aber ebenso ausreichend Raum für die Sensibilisierung der Lernenden im Hinblick auf die phonostilistischen und textsortenspezifischen Besonderheiten der Rede sowie allgemein für die Bewusstmachung der kommunikationspraktischen Relevanz einer akzentfreien, situativ angemessenen Sprechweise geben. Es wird hier nämlich angenommen, dass das phonetisch aufmerksame Sprechen eine der wichtigen Voraussetzungen für die Sicherung von Verständlichkeit sprachlicher Mitteilungen und somit auch für die Vermeidung ungewollter Kommunikationswirkungen, etwa negativer Bewertung der Sprecherperson bzw. deren (Fach-) Kompetenz durch Rezipient(inn)en, darstellt. Davon ausgehend sollte das Erwecken von Aussprachebewusstheit zu einem der übergeordneten Ziele des Fremdsprachenunterrichts schlechthin werden.

\footnotetext{
${ }^{1}$ Im Rahmen der (Auslands-)Germanistik ist die Vermittlung der Zielaussprache gegenwärtig, zumindest in Polen, keine Selbstverständlichkeit (mehr). Zu den wenigen Ausnahmen gehören diesbezüglich die BA-Studiengänge an der Universität Łódź, Universität Wrocław und Universität Rzeszów mit jeweils inhaltlich unterschiedlich konzipierten Lehrprogrammen zur Theorie und Praxis der deutschen Phonetik.
} 
Aussprachebewusstheit ist Gegenstand des vorliegenden Beitrags. Den Ausgangspunkt für weitere Überlegungen bilden Erläuterungen zur Konzeption der Language Awareness im Kontext der Mehrsprachigkeit.

\section{Language Awareness - Sprachbewusstheit}

Das ursprünglich in Großbritannien der 1970er/80er Jahre angelegte Konzept von Language Awareness lässt sich als aufmerksamer und reflektiver Umgang mit Sprache(n) der Umgebung bzw. als Brücke zwischen Mutter-/Erst- und weiteren (Fremd-)Sprachen beschreiben. Mit den sog. Topics der Language Awareness wurden zu Beginn zwei Ziele verfolgt: (1) auf pädagogischer Ebene: die Wahrnehmung, Einbindung und Aufwertung der im Klassenzimmer vorhandenen Erstsprachen und deren Sprecher(innen), und (2) auf kognitiver Ebene: die Vermittlung sprachlicher und metasprachlicher Fähigkeiten sowie Strategien zum Lernen von Sprachen (vgl. Hawkins 1984, 1985). Vor dem Hintergrund der Migration in die BRD und der daraus resultierenden Migrationspolitik fand dieses Konzept auch (zunächst) in die DaM- und DaZ-Didaktik Eingang (ausführlicher dazu vgl. auch Budde 2016:2, Demmig 2016:68f., Oomen-Welke 2016:6f.). Mittlerweile wird der Terminus Language Awareness im deutschsprachigen Raum grundsätzlich mit Sprachbewusstheit bzw. -bewusstsein oder mit Sprachaufmerksamkeit wiedergegeben, wobei aufgrund unterschiedlicher wissenschaftlicher Traditionen und Kontexte ein jeweils unterschiedlicher Fokus gesetzt wird, etwa die metasprachliche und die kognitive Komponente beim Vergleich von Sprachen und im Umgang mit Grammatik ( $\rightarrow$ bei Sprachbewusstheit), ein intuitives in den unbewussten Abläufen verortetes Sprachgefühl im Sinne von Fähigkeit zur Lösung der Sprachproduktionsprobleme ohne bewusste Reflexion ( $\rightarrow$ bei Sprachbewusstsein) und das Moment der Wahrnehmung von Sprachen und Kulturen $(\rightarrow$ bei Sprachaufmerksamkeit) (vgl. u.a. Fujisawa 2017:134 und 137f., auch Spitta 2000:4, Budde 2016:2, Ossner 1989:25f.). ${ }^{2}$ Die hier aufgeführte terminologische Vielfalt wird also durch eine definitorische Unklarheit begleitet, welche wiederum auf die Anwendungsbreite des gesamten Konzepts von Language Awareness (vgl. Demmig 2016:70) bzw. dessen Vagheit oder aber dessen schwere Greifbarkeit (vgl. Fujisawa 2017:134) zurückzuführen ist. Ein Konsens diesbezüglich besteht Knapp-Potthoff (1997:11) zufolge lediglich in der Tatsache, mit Bewusstheit sei eine Reflexionsebene bzw. eine Ebene der mentalen Verarbeitung angesprochen, die über ein rein mechanisches Verhalten und die bloße Verwendung von Sprache als Instrument hinausgehe. Davon wird ebenfalls in den weiteren Ausführungen ausgegangen.

2 Andere in dem hier behandelten Kontext verwendete Termini sind metasprachliches Bewusstsein, Sprachsensibilisierung, Sprachbetrachtung und Sprechen über die Sprache, Sprachgefühl, Spracheinstellung, Sprachinteresse oder Sprachreflexion (vgl. die Übersicht bei Hohm 2005). 
In den Zeiten der überall vorhandenen Mehrsprachigkeit stellt das Konzept der Language Awareness ein tragendes Element der Tertiärsprachendidaktik dar. Das Bewusstwerden verschiedener sprachlicher Phänomene soll dabei vor allem beim Sprachvergleich erfolgen, um die sprachtypologische Nähe bzw. Distanz bestimmen zu können (ausführlicher dazu vgl. u.a. Neuner et al. 2009, Fischer 2003, Hufeisen 1994). Auf die Idee der Language Awareness wird nicht zuletzt auch im „Gemeinsamen europäischen Rahmen: Lernen, lehren, beurteilen“ $(\mathrm{GeR})^{3}$ zurückgegriffen, wo sie als Sensibilisierung bzw. Sensibilität für Sprache und ein allgemeines Sprachbewusstsein bezeichnet wird (dort s. Kap. 5.1.4.1). Die Sensibilität für Sprache und Sprachgebrauch umfasst nach GeR sowohl die Kenntnis als auch das Verstehen der Organisations- und Verwendungsprinzipien von Sprachen und ermöglicht, neue Erfahrungen in einen geordneten Rahmen anzupassen und als Bereicherung zu empfinden. Folglich wird jede weitere Sprache leichter gelernt bzw. verwendet und ,nicht als Bedrohung für das bereits bestehende linguistische System des Lernenden abgelehnt, welches häufig als das normale und ,natürliche angesehen wird" (ebd.). ${ }^{4}$ In diesem Zusammenhang sei mit Eichler/Nold (2007:63) ebenfalls die Fähigkeit zur kontrollierten Anwendung und Beurteilung sprachlicher Normen sowie zum Korrigieren von Verstößen hervorgehoben.

Die hier umrissene Weite der Konzeption von Language Awareness wird in der Aufgliederung ihrer jeweils unterschiedlich gefüllten Domänen besonders deutlich. Im Hinblick darauf unterscheidet Gnutzmann (1997:232f.) zwischen: (1) kognitiver Domäne $(\rightarrow$ die Kenntnis von Gegenständen, die bewusste Wahrnehmung der sprachlichen Umwelt, die geistige Durchdringung des Sprachsystems und das Erkennen von sprachlichen Einheiten, Kontrasten und Regularitäten sowie deren Funktionen und Verwendungen auf verschiedenen sprachlichen Ebenen); (2) Performanz-Domäne ( $\rightarrow$ die effektive Verwendung von Grammatik und Wortschatz in Bezug auf Kommunikationsziele auf Grundlage des Wissens und der sprachlichen Kompetenz); (3) politischer Domäne ( $\rightarrow$ die Erziehung zum kritischen Umgang mit Texten bei besonderem Fokus auf die Beziehung von Sprache und Herrschaft, die Sensibilisierung für das Manipulationspotential von Sprache); (4) sozialer Domäne ( $\rightarrow$ die Beziehung von Sprecher(in) und Hörer(in) und das daraus resultierende Sprachverhalten, der Zusammenhang von Sprachgebrauch und sozialer Schicht oder von Sprache und Geschlecht sowie die unterschiedliche gesellschaftliche Akzeptanz sprachlicher Varietäten und Einzelsprachen) und (5) affektiver Domäne $(\rightarrow$ Einstellungen der Lernenden zu sprachlichen Phänomenen, die Sprachsensibilisierung, die Neugierde und das Interesse an der Sprache, das Wissen um bewusste bzw. unbewusste Reaktion der Rezipienten auf das sprachliche Verhalten sowie Motivation).

3 Vgl. www.goethe.de/z/50/commeuro/5010401.htm.

4 Vgl. https://www.goethe.de/Z/50/commeuro/5010401.htm. 
Abschließend soll an dieser Stelle betont werden, dass die Sprachbewusstheitsprozesse nicht nur, wie man es ursprünglich verstand, im Bereich der Grammatik oder Lexik im Sinne der grammatikalischen bzw. lexikalischen Bewusstheit, sondern auf allen Sprachebenen ablaufen (können). Dies impliziert also einen bewussten Umgang der Lernenden ebenso mit den lautlich-prosodischen Erscheinungen der zu erlernenden Sprache(n) im Sinne der phonetischen/phonologischen bzw. Aussprachebewusstheit, auf die im nächsten Kapitel ausführlicher eingegangen wird.

\section{Phonetische Bewusstheit - phonologische Bewusstheit - Aussprachebewusstheit}

In der englischsprachigen Literatur zur Language Awareness wird grundsätzlich keine scharfe Trennung zwischen phonetischer und phonologischer Bewusstheit unternommen. So steht die phonetische Bewusstheit beispielsweise laut Literaturrecherche von García Lecumberri (2001:238) für „the ability to reflect on and manipulate the sounds and sound system of a language independently of function and meaning [...]"; in ähnlicher Weise fasst Nicholson (1997:53) die phonologische Bewusstheit auf, nämlich als ,the ability to reflect and manipulate the sound components of spoken words". Nach García Lecumberri lassen sich jedoch die beiden Begrifflichkeiten klar voneinander abgrenzen und inhaltlich jeweils unterschiedlich füllen. So kann mit der ersteren auf spezifischere (artikulatorische, akustische und perzeptive) Klangeigenschaften sowie unterschiedliche Realisationen phonologischer Einheiten Bezug genommen werden, die letztere hingegen wäre ausschließlich im Hinblick auf kontrastive (segmentale wie auch suprasegmentale) Einheiten eines Sprachsystems zu verwenden (ausführlicher dazu vgl. auch Piske 2007:155ff.).

Eine derartige terminologische Verwirrung lässt sich nach Fujisawa (2016:133f.) ebenfalls im deutschsprachigen Raum konstatieren. In dem bereits zitierten GeRDokument wird beispielsweise von phonetischem Bewusstsein und dementsprechend von phonetischen Fertigkeiten (dort s. Kap. 5.1.4.2) gesprochen. Gemeint sind damit folgende Fähigkeiten: (1) zur Unterscheidung und zur Produktion ungewohnter Laute und prosodischer Muster; (2) zum Erkennen und zur Verkettung ungewohnter Lautfolgen sowie (3) zur Auflösung eines Lautkontinuums in eine sinnvoll strukturierte Kette phonologischer Elemente. Hinzu kommt auch das Verstehen und Beherrschen der Prozesse der Hörwahrnehmung und der Lautproduktion, die auf das Lernen einer neuen Fremdsprache angewendet werden können. ${ }^{5}$ Diese Fertigkeiten und Fähigkeiten werden jedoch an einer anderen Stelle im GeR (s. Kap. 5.2) unter dem Begriff der phonologischen Kompetenz zusammengefasst.

5 Vgl. https://www.goethe.de/Z/50/commeuro/5010402.htm. 
Hierbei handelt es sich um Kenntnisse und Fertigkeiten der Wahrnehmung und der Produktion in Bezug auf konkrete Aussprachephänomene, ${ }^{6}$ etwa Phoneme mit ihren distinktiven Merkmalen, Allophone, Silbenstruktur, Wort- und Satzakzent, Satzrhythmus, phonetische Reduktionen u.a. Fujisawa (2016:139f.) selbst verwendet konsequenterweise den Terminus der phonetischen Bewusstheit. Er meint damit die Sensibilisierung für die Phonetik einer Sprache mit all ihren Regeln und Funktionen im Sinne einer bewussten Wahrnehmung und betont zugleich die damit einhergehende kognitive Komponente im Sinne eines konkreten Wissens über rezeptive und produktive Eigenschaften einer Sprache sowie die damit zusammenhängenden physiologischen Aspekte. Somit hebt er die phonetische Bewusstheit vom phonetischen Bewusstsein ab, das nach ihm intuitiver Natur ist.

In der deutschsprachigen Fachliteratur zur Tertiärsprachendidaktik ist hingegen weder von phonetischer noch von phonologischer Bewusstheit explizit die Rede. Im Hinblick auf den DaFnE-Unterricht spricht beispielsweise Hirschfeld (2011:210) im Kontext der Phonetik allgemein von sprachlicher Bewusstheit, welche ihr zufolge als einer der positiven Einflussfaktoren auf das fremdsprachliche Lehren und Lernen gilt. Weitere solche Faktoren sind nach Hirschfeld die bereits erworbenen metasprachlichen Kenntnisse (Termini, Internationales Phonetisches Alphabet u.a.), das erweiterte Inventar an feinmotorischen Abläufen (Sprechbewegungen) zur Realisierung von phonetischen Merkmalen oder erweiterte Fähigkeiten und Fertigkeiten in der identifizierenden und differenzierenden Wahrnehmung gesprochener Sprache (das phonologische und phonetische Hören) u.a. (vgl. Hirschfeld 2011:209f.). In demselben Zusammenhang bedient sich wiederum Mehlhorn (2012/2013:187f.) der Begrifflichkeit einer Brücke, die sich auf Grundlage möglicher Ähnlichkeiten zwischen Sprachen ebenfalls im Bereich der Aussprache schaffen lässt. Die Aussprachebrücken betreffen nach Mehlhorn u.a. das Wissen um unterschiedliche Artikulationsweisen ähnlicher Laute und die Erfahrung mit fremden Artikulationsbewegungen sowie die Handhabung von Begriffen einschließlich der dahinter stehenden Konzepte. Zu betonen ist hierbei nicht zuletzt die Bewusstmachung einer automatischen Übertragung muttersprachlicher Besonderheiten in die Zielaussprache im Sinne des fremden Akzents (vgl. Mehlhorn 2012/2013:179f.). Im Hinblick darauf weisen Mehlhorn/Trouvain (2007) auf die Relevanz von $\mathrm{L}_{1}-\mathrm{L}_{2}$-Vergleichen, etwa in einem kontrastiven Phonetikunterricht z.B. mit Fokus auf die Prosodie, hin.

Der oben aufgezeigten terminologisch-definitorischen Uneinheitlichkeit liegt wohl die gegenstandsbedingte Verwandtschaft von Phonetik und Phonologie zugrunde. Beide Teildisziplinen setzen sich nämlich ihrem Wesen nach mit den lautlichen und prosodischen Phänomenen der Sprache auseinander, auch wenn sie es aus unterschiedlichen Blickwinkeln tun: Während im Rahmen der Phonetik konkrete (artikulatorische, auditive und akustische) Eigenschaften der Sprachlaute

${ }^{6}$ Vgl. https://www.goethe.de/Z/50/commeuro/5020104.htm. 
sowie lautübergreifende (prosodische) Erscheinungen der gesprochenen Sprache untersucht werden, so gilt Phonologie als Lehre über deren Funktion(en) in einem konkreten Sprachsystem. Es war allerdings nicht das Anliegen dieses Beitrags, über den höheren Rang des einen oder des anderen Mutterfachgebiets bzw. der einen oder der anderen Begrifflichkeit - der phonetischen oder der phonologischen Bewusstheit $-\mathrm{zu}$ diskutieren und $\mathrm{zu}$ entscheiden. Um vielmehr von terminologischen Fragen absehen zu können, wurde hier der Terminus der Aussprachebewusstheit eingeführt und als quasi Oberbegriff verwendet. Gemeint ist damit allgemein die Bewusstheit für Aussprachephänomene im weiteren Verständnis - für die Sprachlaute und die prosodischen Erscheinungen der Rede einschließlich ihrer Distinktivität und anderer möglicher, darunter situativ/phonostilistisch geprägter Funktionen bzw. Gebrauchsweisen.

Der bewusste Umgang mit den Aussprachephänomenen sollte in der Fremdsprachen-Lehr- und Lernpraxis wie auch in der kommunikativen Wirklichkeit dem Konzept der Language Awareness nach optimalerweise in unterschiedlichen Domänen gleichzeitig erfolgen, etwa im Sinne von Gnutzmann (1997) - in der Domäne der Kognition, der Performanz, der Emotion und der sozialen Beziehungen mit jeweils unterschiedlichem Fokus. Dementsprechend sollten diesbezüglich im Einzelnen folgende Aspekte besonders relevant werden:

1. in der Domäne der Kognition

a) das Wissen um jeweils vorhandene phonetisch-phonologische Erscheinungen bzw. Regularitäten:

- das Lautsystem und prosodische Phänomene (Wort- und Satzbetonung, Melodisierung, Pausen, Sprechrhythmus) und ihre distinktiven Eigenschaften,

- die Lautdistribution und Silbenstruktur,

- Laut-Graphem-Beziehungen sowie das Wissen um ihre Funktionen und Verwendungen im System der zu erlernenden Sprache(n) wie auch in der Ausgangssprache;

b) die Hörwahrnehmung, Erkennung und Verarbeitung sprecherischer Phänomene, d.h. die Fähigkeit zum phonologischen und phonetischen Hören, etwa in Bezug auf:

- die Gliederung von Wörtern in Silben,

- die Erkennung von Minimalpaaren,

- die Identifizierung und Differenzierung von Sprachlauten und von prosodischen Abläufen;

c) die Wahrnehmung von verschiedenen Sprechstilen bzw. phonostilistischen Variationen $(\rightarrow$ die Unterscheidung zwischen emotionalem und sachlich-neutralem Sprechen) sowie das Wissen um kommunikative Funktionen dieser Variationen;

2. in der Domäne der Performanz

a) die der Standardaussprache angemessene Realisierung der einzelnen Sprachlaute ( $\rightarrow$ artikulatorisch-motorische Automatismen); 
b) die situativ und textsortenangemessene Realisierung der jeweiligen prosodischen Phänomene $(\rightarrow$ Automatismen im Bereich der Prosodie sowie die Sprechflüssigkeit);

c) die Fähigkeit zur phonostilistischen Variation $(\rightarrow$ etwa zum emotionalen vs. sachlich-neutralen Sprechen);

3. in der Domäne der Emotion

a) die Sensibilisierung der Lernenden für die fremdsprachlichen Klänge $(\rightarrow$ die Beeinflussung ihrer Einstellung zu neuen Sprachklängen und deren Akzep$\operatorname{tanz}$ );

b) das Wecken von Neugierde bei den Lernenden auf die stimmlich-prosodische Vielfalt in der Zielsprache in situativ unterschiedlichen sozialen Interaktionen $(\rightarrow$ phonostilistische/situative Variation);

c) die Förderung der Lernmotivation im Bereich der Zielaussprache etwa bezüglich der Eliminierung des fremden Akzents und der Sicherung der phonetischen Verständlichkeit o.ä.;

4. in der Domäne der sozialen Beziehungen

a) die Erreichung kommunikativer Absichten u.a. mittels sprachlich-phonetischer Ausdrucksmittel;

b) mögliche ungewollte Kommunikationswirkungen, z.B. die negative Beurteilung der Sprecherperson, ggf. deren (Fach-)Kompetenz, bzw. ihre gesellschaftliche Nicht-Akzeptanz aufgrund des fremden Akzents und/oder aufgrund des unangemessenen Einsatzes der Prosodie in ihrer pragmatischen bzw. rhetorischen Funktion.

\section{Fazit}

Die in dem vorliegenden Beitrag vorgeschlagene Betrachtung von Aussprachebewusstheit geht grundsätzlich mit der kommunikativ-pragmatischen Sprachauffassung einher, welche wiederum die Verankerung sprachlicher Geschehen in sozialen Interaktionen schlechthin impliziert. Im Hinblick darauf soll abschließend vor allem die folgende Tatsache nachdrücklich betont werden: Bei der Vermittlung einer angemessenen Zielaussprache sollte nicht nur die Sensibilisierung der Lernenden für die jeweils gegebenen Klangunterschiede zwischen der Ausgangs- und Zielsprache angestrebt werden, d.h. es sollten nicht nur konkrete Wissensbestände im Bereich der Phonetik und Phonologie vermittelt und/oder konkrete auditive sowie motorisch-artikulatorische Fähigkeiten und Fertigkeiten als Voraussetzung fürs akzentfreie Sprechen trainiert werden. Im Rahmen einer kommunikativ angelegten Aussprachevermittlung sind darüber hinaus auch konkrete kommunikativ relevante Aspekte, etwa die Rolle der phonetischen Mittel beim Ausdruck von Emotionen bzw. die bewusst anzustrebende Sachlichkeit in rhetorischen Sprechsituationen, zu problematisieren. Schließlich sollten den Lernenden ebenfalls mög- 
liche negative Kommunikationswirkungen infolge der Beeinträchtigung phonetischer Verständlichkeit vor Augen geführt werden.

Zur Entwicklung von Aussprachebewusstheit können in der Lehrpraxis insgesamt unterschiedliche, im Phonetikunterricht mittlerweile etablierte Methoden, u.a. systematische Vergleiche von Lautsystemen, die Visualisierung der Lautartikulation oder der prosodischen Abläufe (von Sprechrhythmus, Betonungen, Melodisierung etc.), herangezogen werden. Diesbezüglich lassen sich ebenso konkrete Arbeitsweisen aus dem Bereich der Rhetorik anwenden, etwa das Beobachten fremder und eigener Sprechleistungen zur Bewusstmachung möglicher Kommunikationswirkungen oder der Stichwortzettel zur Erreichung von sprachlich-phonetischen Automatismen und Sprechflüssigkeit. Ausführlicher soll darauf an einer anderen Stelle eingegangen werden.

\section{Literaturverzeichnis}

ANDRESEN Helga, 1985, Schriftspracherwerb und die Entstehung von Sprachbewußtheit, Opladen. BudDe Monika, 2016, Mehrsprachigkeit - Language Awareness - Sprachbewusstheit. Eine Einführung, in: Zeitschrift für Interkulturellen Fremdsprachenunterricht 21/2, S. 1-4, http:// tujournals.ulb.tu-darmstadt.de/index.php/zif/article/view/808/809. Zugriff am 19.1.2021.

Demmig Silvia, 2016, Language Awareness und Deutsch als Fremdsprache, in: Zeitschrift für Interkulturellen Fremdsprachenunterricht 21/2, S. 68-75, http://tujournals.ulb.tu-darmstadt.de/ index.php/zif/article/view/814/815. Zugriff am 19.1.2021.

EICHLER Wolfgang / Nold Günter, 2007, Sprachbewusstheit, in: Beck B./Klieme E. (Hrsg.), Sprachliche Kompetenzen Konzepte und Messung. DESI-Studie (Deutsch Englisch Schülerleistungen International), Weinheim/Basel, S. 63-82, http://www.pedocs.de/volltexte/2010/3242/ pdf/Eichler_Sprachbewusstheit_2007_D_A.pdf. Zugriff am 19.1.2021.

Fischer Sylvia, 2003, Awareness- und Immersionstrategien im universitären DaF-Unterricht, in: Info DaF 30/5, S. 428-438.

FujISAWA David, 2017, Language Awareness und Phonetik im (Deutsch als) Fremdsprachunterricht, in: Bose I./Hannken-Illjes K./Hirschfeld U./Neuber B. (Hrsg.), Forschung und Didaktik der Sprechwissenschaft. Aktuelle Beiträge. SSP 11, Berlin, S. 133-144.

García Lecumberri Maria Luisa, 2001, Phonetic awareness, in: Lasagabaster D./Sierra J.M. (Hrsg.), Language Awareness in the Foreign Language Classroom, Leioa, S. 237-251.

Gnutzmann Claus, 1997, Language Awareness. Geschichte, Grundlagen, Anwendung, in: Praxis des neusprachlichen Unterrichts 3/97, S. 227-236.

Hawkins Eric, 1984, Awareness of Language. An Introduction, Cambridge.

Hawkins Eric, 1985, Awareness of Language. Series, Cambridge.

HirschFeld Ursula, 2011, Aussprachetraining in Deutsch als 2. Fremdsprache (nach Englisch), in: Barkowski H./Demmig S./Funk H./Würz U. (Hrsg.), Deutsch bewegt. Entwicklungen in der Auslandsgermanistik und Deutsch als Fremd- und Zweitsprache (Dokumentation der Plenarvorträge der XIV. Internationalen Tagung der Deutschlehrerinnen und Deutschlehrer IDT Jena-Weimar 2009), Hohengehren, S. 207-220.

Нонм Michael, 2005, Zum Zusammenhang von Sprachbewusstheit, Lesekompetenz und Textverstehen. Historische, fachdidaktische und unterrichtspraktische Aspekte der Problematik (unveröff. Dissertation), Würzburg, http://opus.bibliothek.uni-wuerzburg.de/opus4-wuerzburg/ frontdoor/deliver/index/docId/1653/file/hohm.pdf. Zugriff am 19.1.2021. 
Hufeisen Britta, 1999, Deutsch als zweite Fremdsprache. Einleitung zum Themenheft, in: Fremdsprache Deutsch. Zeitschrift für die Praxis des Deutschunterrichts 20, S. 4-6.

Knapp-Potthoff Annelie, 1997, Sprach(lern)bewußtheit im Kontext, in: Fremdsprachen Lehren und Lernen 26, S. 9-23.

MeHLHORN Grit, 2012/2013, Lebenslanges Lernen und Mehrsprachigkeit: Deutsch als Fremdsprache nach Englisch im russischsprachigen Kontext, in: Das Wort. Germanistisches Jahrbuch Russland, S. 177-193, http://wort.daad.ru/wort2012/18+Mehlhorn.pdf. Zugriff am 19.1.2021.

Mehlhorn Grit / Trouvain Jürgen, 2007, Sensibilisierung von Lernenden für fremdsprachliche Prosodie, in: Zeitschrift für interkulturellen Fremdsprachenunterricht 12, S. 1-25, http://tujournals.ulb.tu-darmstadt.de/index.php/zif/article/view/304/296. Zugriff am 19.1.2021.

Neuland Eva, 2002, Sprachbewusstsein - eine zentrale Kategorie für den Sprachunterricht, in: Der Deutschunterricht 3/02, S. 4-10.

Neuner Gerhard / Hufeisen Brita / Kursiša Anta / Marx Nicole / Koithan Ute / Erlenwein Sabine, 2009, Deutsch als zweite Fremdsprache. Fernstudieneinheit 26, Berlin/München/ Zürich/New York.

NichOLSON Tom, 1997, Phonological awareness and learning to read, in: van Lier L./Corson D. (Hrsg.), Encyclopedia of Language and Education, Volume 6. Knowledge about Language, Dordrecht, S. 53-62.

Oomen-Welke Ingelore, 2016, Mehrsprachigkeit - Language Awareness - Sprachbewusstheit. Eine persönliche Einführung, in: Zeitschrift für interkulturellen Fremdsprachenunterricht 21, S. 1-12, http://tujournals.ulb.tu-darmstadt.de/index.php/zif/article/view/809. Zugriff am 19.1.2021.

OSSNER Jakob, 1989, Sprachthematisierung - Sprachaufmerksamkeit - Sprachwissen, in: OBST. Sprachbewußtheit und Schulgrammatik 40, S. 25-38.

PIske Thorsten, 2008, Phonetic Awareness, Phonetic Sensitivity and the Second Language Learner, in: Hornbereger N.H. (Hrsg.), Encyclopedia of Language and Education, Volume 5, Boston, S. $155-166$.

SpitTa Gudrun, 2000, Sind Sprachbewusstheit und Sprachbewusstsein dasselbe? Oder Gedanken zu einer vernachlässigten Differenzierung, in: Deutschdidaktische Perspektiven. Eine Schriftenreihe des Studiengangs Primarstufe an der Universität Bremen im Fachbereich 12: Bildungs- und Erziehungswissenschaften, Universität Bremen, http://docplayer.org/37526156Deutschdidaktische-perspektiven.html. Zugriff am 19.1.2021.

\section{Some thoughts on the awareness of pronunciation in the context of foreign language teaching}

Multilingualism at the European or worldwide level is related to the need for changing the perception of foreign language teaching and learning. As a result, the concept of language awareness has been given a high priority. It implies the reflection on the language elements and their functions language comparisons on the one hand, and the interdisciplinary sensitivisation for languages with the inclusion of language curiosity, etc. on the other. At the same time, the concept of language awareness plays a significant role in the field of tertiary language didactics, such as teaching German after English. With that in mind, this article presents in-depth reflections about the consciousness of pronunciation aspects and their multidimensional character.

Keywords: phonetic/phonological/pronunciation awareness, teaching pronunciation, German as a foreign language. 